\title{
Estudio de la citotoxicidad de películas nanohíbridas con matriz de poliestireno reciclado
}

\section{Study of the cytotoxicity of nanohybrid films with recycled polystyrene matrix}

\author{
Alejandra Blanco-Hernández, ${ }^{*}$ René García Contreras, ${ }^{* *}$ \\ Paloma Serrano Díaz** y Genoveva Hernández-Padrón***
}

\begin{abstract}
The present work shows the development of hybrid films adapting the sol-gel process with an adequate control in the design of the experimental conditions in order to incorporate silica nanoparticles to a recycled polystyrene polymer matrix (PSR). For a better incorporation between the silica and the PSR it was necessary to functionalize the recycled polystyrene with carboxyl groups of abietic acid (PSRF). The PSR used for the preparation of nanohybrid films was obtained from fast food packaging products. The cytotoxicity of the PSR films without substrate was evaluated, showing viability to be used as protective films on glass substrates, this is of high relevance due to the fact that they do not present a health risk. The hybrid materials were characterized by infrared spectroscopy. Also, mechanical and thermal properties of the hybrid materials developed were evaluated. The glass transition temperature increased for functionalized hybrid materials (HPSF) and recycled-functionalized hybrid materials (HPSRF) due to the presence of abietic acid and silica. All materials increased their contact angle and hydrophobic capacity compared to that of the uncoated substrate. The incorporation of silica particles within the polymer matrix shows interesting improvements in the evaluated properties compared to the hybrid materials obtained from commercial polystyrene (PS). Derived from these results, the materials developed in this work are suggested for applications such as coatings on glass substrates with self-cleaning properties in terms of hydrophobicity and UVA radiation protection, which are of great technological relevance today.
\end{abstract}

KEYWORDS: recycled polystyrene, hybrid films, cytotoxicity, hydrophobicity.

RESUMEN: En el presente trabajo se muestra el desarrollo de películas híbridas adaptando el proceso sol-gel con un control adecuado en el diseño de las condiciones experimentales con el fin de incorporar nanopartículas de sílice a una matriz polimérica de poliestireno reciclado (PSR). Para una mejor incorporación entre la sílice y el PSR fue necesario funcionalizar el poliestireno reciclado con grupos carboxilo de ácido abiético (PSRF). El PSR utilizado para la preparación de películas nanohíbridas se obtuvo a partir de productos de empaques de comida rápida. Se evaluó la citotoxicidad de las películas de PSR sin sustrato, mostrando viabilidad de utilizarse como películas

Recibido: 21 de noviembre de 2017.

Aceptado: 15 de abril de 2018.

* Instituto Politécnico Nacional-Zacatenco, Escuela Superior de Ingeniería Química e Industrias Extractivas, Ciudad de México, México.

** Universidad Nacional Autónoma de México, Escuela Nacional de Estudios Superiores Unidad León, Guanajuato, México.

*** Universidad Nacional Autónoma de México, Centro de Física Aplicada y Tecnología Avanzada, Departamento de Nanotecnología Campus Juriquilla, Querétaro, México.

Autora para correspondencia: (genoveva@unam.mx). 
Mundo Nano | ARTículos | www.mundonano.unam.mx

11(21), 61-71, julio-diciembre 2018 | http://dx.doi.org/10.22201/ceiich.24485691e.2018.21.62567

Alejandra Blanco-Hernández, René García Contreras, Paloma Serrano Díaz

protectoras en sustratos de vidrio, lo anterior es de alta relevancia debido a que no presentan riesgo para la salud. Los materiales híbridos fueron caracterizados por las espectroscopías infrarrojo. También se evaluaron propiedades mecánicas y térmicas de los materiales híbridos desarrollados. La temperatura de transición vítrea incrementó para los materiales híbridos funcionalizados (HPSF) y los materiales híbridos reciclados-funcionalizados (HPSRF) debido a la presencia del ácido abiético y la sílice. Todos los materiales incrementaron su ángulo de contacto y la capacidad hidrófoba comparando con el del sustrato sin recubrir. La incorporación de partículas de sílice dentro de la matriz polimérica muestra mejoras interesantes en las propiedades evaluadas en comparación con los materiales híbridos obtenidos a partir de poliestireno comercial (PS). Derivado de estos resultados, los materiales desarrollados en este trabajo se sugieren para aplicaciones como recubrimientos en sustratos de vidrio con propiedades de autolimpieza en términos de hidrofobicidad y protección a la radiación uva que hoy en día son de gran relevancia tecnológica.

PALABRAS CLAVE: poliestireno reciclado, películas híbridas, citotoxicidad, hidrofobicidad.

\section{Introducción}

La búsqueda de nuevas alternativas a la biodegradabilidad, el reciclado o la reutilización de materiales poliméricos abre hoy en día una línea de investigación muy amplia e importante (Maharana y Mohanty, 2014). La preocupación que nos atañe a los seres humanos es proteger o remediar el medio ambiente que hemos ido deteriorando (Ilgenenfritz, 1975; Sanchez, et.al, 2011; Gerasin, et al., 2013). Es por esa razón que en este trabajo se propone la reutilización de poliestireno cristal que desechamos cotidianamente a través de contenedores transparentes de alimentos o bebidas.

La propuesta del presente trabajo es reciclar poliestireno como recubrimiento de ventanas con características repelentes al agua, antiempañantes o autolimpiables pues se sabe que los polímeros como el LDPE, HDPE, PS, PET, entre otros, polímeros son utilizados en este tipo de aplicaciones (Stevenson, White, 2012; Borsoia et al., 2014; Hwang, et al., 2008). Primeramente se modificó la superficie del PS y PSR con un grupo hidrofóbico, con el fin de mejorar las características hidrófobas del polímero. Asimismo, se incorporaron nanopartículas de sílice en el PS y PSR funcionalizado (PSF y PSRF), combinando las características del poliestireno y de la sílice a través del proceso sol-gel (Hossein et al., 2011; Zou et al., 2008; Hernández-Padrón et al., 2003), lo que promete un mejor desempeño hidrofóbico del recubrimiento. En trabajos anteriores se han observado propiedades de alta resistencia termo-mecánica, y anticorrosión en sustratos metálicos (Hernández-Padrón et al., 2010, 2015). La incorporación de estas nanopartículas se realiza mediante el proceso solgel. Es menester mencionar que a partir de las condciones iniciales en el proceso sol-gel es posible manipular las características químicas y físicas de los nuevos materiales a niveles desde moleculares hasta nanométricos (Kotoky y Dolui, 2006; BaoLi y DuXin, 2007). Las propiedades de estos materiales no sólo dependen de sus componentes individuales sino también de su morfología y propiedades interfaciales. Esto dirige a prever todas las propiedades finales del material. Actualmente, la idea para producir nuevos materiales es 
predecir las propiedades antes de elaborar un material, este hecho conduce a seguir ampliando conocimientos en el área de los materiales híbridos.

En estos nuevos nanomateriales la incorporación de los soles de sílice sobre la superficie de la matriz polimérica da por resultado nuevas y muy interesantes propiedades, las cuales fueron evaluadas mediante diferentes técnicas analíticas. Con base en los resultados obtenidos mediante la caracterización de los materiales desarrollados éstos pueden ser de relevancia tecnológica, debido a que, con un adecuado control en el diseño de las condiciones experimentales, se puede desarrollar una amplia gama de estos nuevos materiales sin generar efectos colaterales a la salud.

Una medida importante para medir la repelencia (hidrofobicidad) al agua en el recubrimiento es la disminución del ángulo de contacto en la interfase con el sustrato. La hidrofobicidad del recubrimiento fue evaluada a través de la medición del ángulo de contacto, tomando el criterio del ángulo de contacto formado, siendo menor a $40^{\circ} \mathrm{C}$ para una superficie hidrofílica, y un ángulo de contacto mayor a $70^{\circ} \mathrm{C}$ se considera como superficie hidrofóbica (Howarter et al., 2008).

Todos los materiales desarrollados mostraron propiedades de hidrofobicidad, antiempañamiento y transparencia, así como propiedades mecánicas tales como resistencia a la flexión. Por otra parte, las muestras no mostraron citotoxicidad con un rango de viabilidad de $75-103 \%$. Lo anterior permite abrir una nueva alternativa viable para la reutilización del poliestireno cristal, generando recubrimientos de alta calidad en cuanto a propiedades ópticas y mecánicas.

\section{Procedimiento experimental}

\section{Materiales}

Todas las muestras se prepararon usando Tetraethyl orthosilicate (TEOS de Aldrich Chem.), agua destilada, tolueno grado reactivo (J. T. Baker), poliestireno cristal comercial (Resirene Co.) y poliestireno reciclado de contenedores de bebidas frías.

\section{Funcionalización de poliestireno comercial y reciclado (PSF y PSRF)}

Cada uno de los poliestirenos se funcionalizaron con grupos carboxilo. El poliestireno correspondiente se mezcló con ácido abiético (AA) en solución de tolueno en una relación peso de PS o PSR con AA de 0.1, usando peróxido de benzoilo en una relación molar de $1 \times 10^{-2}$ respecto al AA, la mezcla se mantuvo en agitación mecánicamente durante 1h (Hernández-Padrón et al., 2014).

\section{Preparación de materiales híbridos HPSR y HPSRF}

El material híbrido de cada uno de los poliestirenos funcionlizados (PSF y PSRF) se preparó a partir de soluciones anteriores. Por separado, TEOS con tolueno se mezcló con agitación magnética por 10 minutos. Posteriormente se 
mezcló con la solución de poliestireno funcionalizado correspondiente, la solución se incorpora con agitación vigorosa. La solución resultante se agitó y se dejó en reflujo durante $1 \mathrm{~h}$.

\section{Caracterización}

La caracterización de los materiales resultantes se llevó a cabo por espectroscopia FTIR (Fourier-transform infrared spectroscopy) El análisis infrarrojo se realizó en un espectrómetro Bruker Vector 33, utilizando la técnica de reflectancia total atenuada (ATR) utilizando un cristal de diamante y 32 escaneos.

La morfología de los materiales híbridos se observó mediante microscopía electrónica de barrido (SEM). Las observaciones se llevaron a cabo en un microscopio de exploración Jeol JSM-5200. Las muestras se recubrieron con carbono por evaporación al vacío.

El análisis térmico TGA / DTA se llevó a cabo en un instrumento DuPont 951 operado en una atmósfera de aire a una velocidad de $10 \mathrm{~K} \mathrm{~min}^{-1}$.

\section{Cultivo de celular}

Los fibroblastos gingivales humanos (HGF) se obtuvieron de una biopsia de tejido gingival durante la extracción de tercer molar de un paciente de 18 años de edad, con previa autorización del consentimiento informado. El proyecto fue autorizado por el Comité de Bioética de ENES Unidad León, UNAM. El tejido fue almacenado en solución salina buffer de fosfato (PBS) y antibióticos al $2 \%$ ( $100 \mathrm{UI} / \mathrm{ml}$ de penicilina $\mathrm{G}$ y $100 \mathrm{mg} / \mathrm{ml}$ de sulfato de estreptomicina), se lavó dos veces con PBS y el tejido fue dividido en explantes de $1 \times 1 \mathrm{~mm}$, aproximadamente. El tejido fue suspendido en medio $\alpha$-MEM (Gibco) suplementado con el 20\% de suero fetal bovino (FBS, Gibco), 1\% de antibióticos y $1 \%$ de glunamina (Glutamax, Gibco) de estreptomicina (Gibco) e incubadas a $37^{\circ} \mathrm{C}$ con una atmósfera de $5 \%$ de $\mathrm{CO}_{2}$ y una humedad del $95 \%$ durante dos semanas hasta observar un crecimiento celular exponencial y con cambio del medio de cultivo cada tercer día. Los HGF tiene una esperanza de vida in vitro de aproximadamente de 40 PDL (Nivel doble de población). Las células se desprendieron enzimáticamente del plato de cultivo con $0.25 \%$ de tripsina-EDTA $0.025 \%-2 \mathrm{Na}$ (Gibco) para cada experimento. Después de que el cultivo celular primario fue establecido, los experimentos se llevaron a cabo usando medio DMEM complementado con $10 \%$ de FBS, antibiótico al $2 \%$ y glutamina al $2 \%$.

\section{Ensayo de actividad citotóxica}

Células HGF fueron inoculadas a una densidad de $2 \times 10^{5}$ células $/ \mathrm{ml}$ en un plato de 96 -pocillos e incubadas durante 48 horas a $37^{\circ} \mathrm{C}$ con $5 \%$ de $\mathrm{CO}_{2}$ y 95\% de humedad relativa. Las películas fueron inoculadas (1-10 muestras) en cada uno de los pocillos y se agregó medio de cultivo suplementado fresco e incubadas por 24 horas. El número de células viables fue determinado por el método MTT. Brevemente, $0.2 \mathrm{mg} / \mathrm{ml}$ del reactivo MTT (Sigma) fue 
mezclado en DMEM $+10 \%$ FBS y se incubó durante 4 horas a $37^{\circ} \mathrm{C}$ con $5 \%$ de $\mathrm{CO}_{2}$ y $95 \%$ de humedad relativa. El formazán fue disuelto completamente con sulfóxido de dimetil (DMSO, Karal) y analizado en un lector de microplaca (ThermoFisher Scientific) a $570 \mathrm{~nm}$. Se utilizó como control pocillos de cultivo con células. Los ensayos se realizaron por triplicado a partir de tres experimentos independientes con base a la NOM ISO 10993 (Biological evaluation of medical devices - Part 5: Tests for in vitro cytotoxicity) donde la interpretación de la viabilidad celular y citotoxicidad resultante fue considerada de la siguiente manera: No citotóxico: 100-75\%, ligeramente citotóxico: $74-50 \%$, moderadamente citotóxico: $49-25 \%$, y, extremadamente citotóxico: $24-0 \%$.

\section{Análisis estadístico}

Para el ensayo de citotoxicidad se calculó la media, la desviación estándar y el porcentaje. Todos los datos fueron sometidos a pruebas de normalidad Shapiro-Wilks, pueba de ANOVA pos hoc de Tukey. La significancia estadística fue considerada con un $\mathrm{p}<0.05$ y un intervalo de confianza del $95 \%$.

El ángulo de contacto fue medido en el recubrimiento del material preparado sobre el vidrio de superficie colocando una gota de agua $(1 \mu l)$ en la superficie, luego se tomó una imagen en la interfaz sólido-líquido con una cámara Cannon 70D. Estas imágenes se transformaron en escala de grises y se analizaron con el programa matemática. Las medidas se hicieron con cinco replicas.

\section{Resultados y discusión}

La figura 1 muestra los espectros de infrarrojo de las muestras: a) PSR, b) PSRF, C) PS y d) PSF, donde se puede observar la funcionalización del poliestireno tanto comercial como el reciclado con grupos carboxílicos debido a la presencia de las bandas de absorción en 1,730 y $1,226 \mathrm{~cm}^{-1}$ del grupo funcional $(C=O)$, esta última señal es atribuida a la vibración del grupo éster $\mathrm{COC}$ (Colthup, Wiberley, 1990).

En la figura 2, se muestran los espectros de infrarrojo de los híbridos (a) HPRS y (b) HPSRF en donde se observan las bandas de absorción en la región entre 2,800 y $3,000 \mathrm{~cm}^{-1}$, que están asociados con las bandas de absorción características de poliestireno (Colthup, Wiberley, 1990). En los grupos éster del carbonilo se perciben $1,730 \mathrm{~cm}^{-1}$ y son evidencia de la reacción entre grupos $\mathrm{OH}$ (pertenecientes a grupos carboxílicos del PSF) y grupos silanol en la superficie de las partículas de sílice (Colthup, Wiberley, 1990). Esta reacción se ve favorecida por la alta electronegatividad del oxígeno y el par solitario de electrones fácilmente disponible del grupo carbonilo, y también por la naturaleza ácida de los grupos $\mathrm{SiOH}$. De esta manera, las cadenas de polímero de PSF se unen a grupos silanol en la superficie de microesferas de $\mathrm{SiO}_{2}$. También es posible observar que las bandas correspondientes a este 
Mundo Nano | ARTículos | www.mundonano.unam.mx

11(21), 61-71, julio-diciembre 2018 | http://dx.doi.org/10.22201/ceiich.24485691e.2018.21.62567

Alejandra Blanco-Hernández, René García Contreras, Paloma Serrano Díaz

FIGURA 1. Espectros de FT-IR (a) PSR, (b) PSRF, (c) PS y (d) PSF.

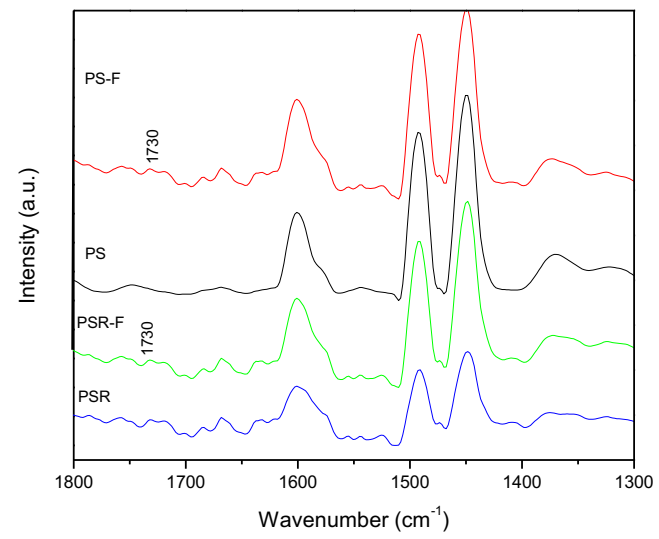

Fuente: Elaboración de los autores.

FIGURA 2. Espectro de infrarrojo de los híbridos (a) HPRS y (b) HPSRF.

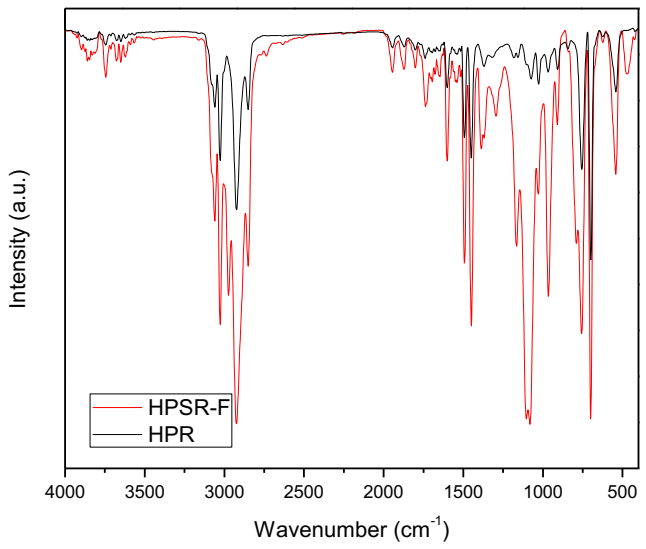

Fuente: Elaboración de los autores.

material híbrido en el intervalo de $1,200-900 \mathrm{~cm}^{-1}$ se vuelven más anchas (con respecto a la misma señal proporcionada por el espectro de PSR) debido a la interacción entre grupos del polímero y oH grupos de las partículas de sílice.

De los termogramas TGA de los materiales preparados, se puede apreciar a partir de este gráfico que la creciente presencia de sílice y la funcionalización mejora la estabilidad térmica de los materiales híbridos, figura 3.

También se realizó un estudio fotográfico SEM de materiales híbridos y no híbridos. Estos materiales caracterizados muestran algunas observaciones importantes sobre las morfologías así como con respecto a algunas otras propiedades ópticas o interesantes de estos sólidos. En la figura 4, se 
FIGURA 3. Termograma TGA de los materiales preparados.

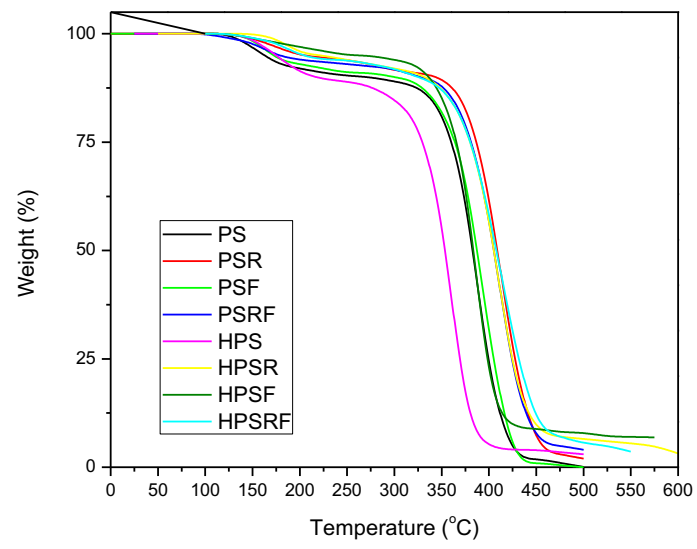

Fuente: Elaboración de los autores.

puede observar que el HPS presenta una segregación de cúmulos de partículas de sílice, es decir, la muestra no es homogénea, en el caso de HPSF, la muestra se puede observar muy homogénea en su superficie, no presenta cúmulos de partículas de sílice, lo cual se puede atribuir a una buena interacción del TEOS con el poliestireno funcionalizado.

En la figura 5, se muestran las microfotografías de los materiales híbridos del poliestireno reciclado, y puede observarse una mayor interacción entre las partículas de sílice y el poliestireno reciclado funcionalizado. Como el caso anterior se puede suponer que el ácido abiético permite que las partículas de sílice puedan dispersarse en la matriz polimérica formando una superficie libre de cúmulos. Además, se puede observar que el HPSR no presenta cúmulos grandes como en el caso del HPS (figura 4a).

FIGURA 4. Micrografías SEM de (a) HPS y (b) HPSF.

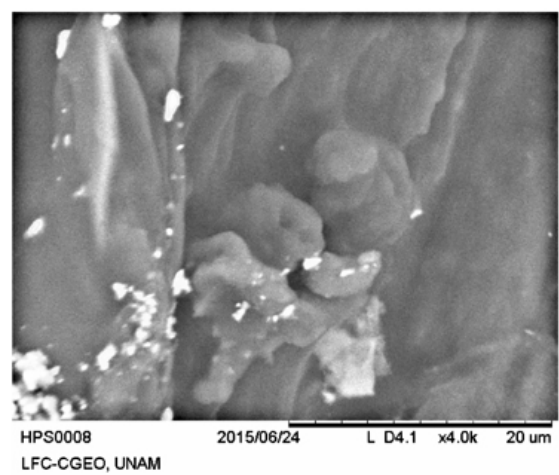

a)

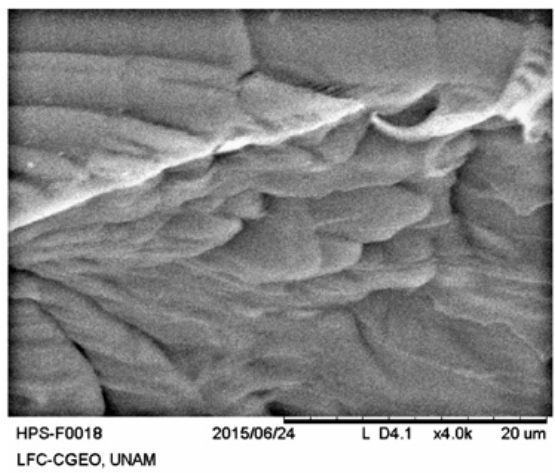

b)

Fuente: Elaboración de los autores. 
Mundo Nano | ARTículos | www.mundonano.unam.mx

11(21), 61-71, julio-diciembre 2018 | http:// dx.doi.org/10.22201/ceiich.24485691e.2018.21.62567

Alejandra Blanco-Hernández, René García Contreras, Paloma Serrano Díaz

FIGURA 5. Micrografias SEM de (a) HPSR y (b) HPSRF.

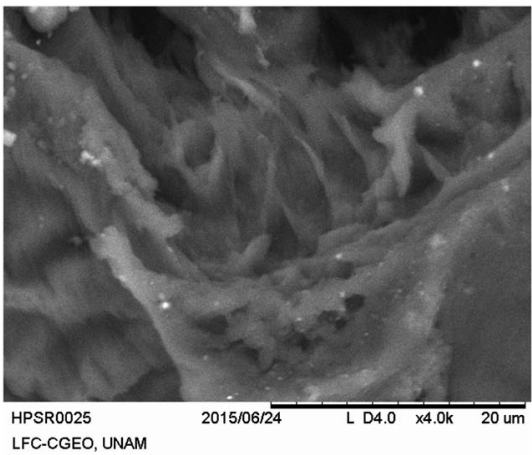

a)

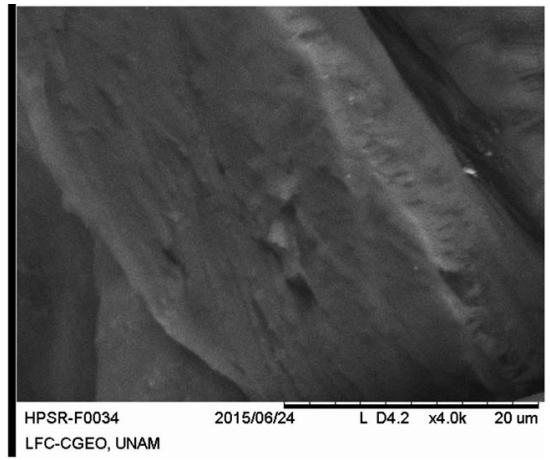

b)

Fuente: Elaboración de los autores.

La idea general del estudio de la morfología de la superficie de los materiales híbridos es determinar la homogeneidad a partir de la dispersión de las partículas de sílice, porque esta característica es importante para determinar la transparencia, traslucidez u opacidad de las películas (HernándezPadrón, et al., 2003), ya que la aplicación final de los híbridos es producir películas de recubrimiento sobre vidrio.

En la figura 6, se muestran los resultados de citotoxicidad donde se puede observar que las muestras de poliestireno reciclado y sus híbridos no mostraron citotoxicidad con una rango de viabilidad de 75-103\% (0.5261.021 absorbancia), con una ligera disminución de la viabilidad en las muestras poliestireno comercial (HPS, PSF y HPSF).

FIGURA 6. Actividad citotóxica de sustratos PSR, PSRF, HPSR, HPSRF, PS, PSF, HPS e HPSF, CONT (HGF control). Fibroblastos gingivales humanos (HGF) fueron inoculados en platos de 96-pocillos junto con las películas. La viabilidad celular fue determinada por el ensayo de MTT a $570 \mathrm{~nm}$. El rango de viabilidad celular fue de 75-103\% (0.526-1.021 absorbancia) * $\mathrm{P}<0.05$, ANOVA pos hoc de Tukey.

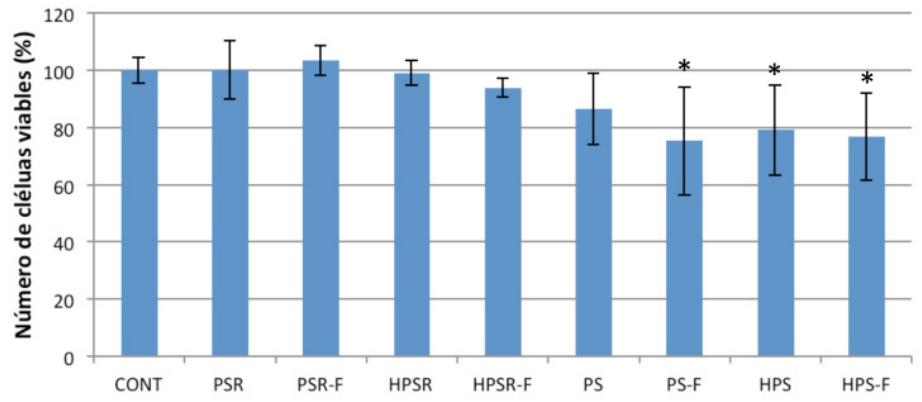

Fuente: Elaboración de los autores.

Sustratos 
El orden de la viabilidad celular correspondió de la siguiente manera: Muestra $\mathrm{PSRF}<\mathrm{PSR}<\mathrm{HPSR}<\mathrm{HPSRF}<\mathrm{PS}<\mathrm{HPS}<\mathrm{HPSF}$ y PSF. Las muestras PSF, HPS y HPSF redujeron significativamente la viabilidad celular $(\mathrm{p}<0.05)$.

En la tabla 1, se muestran los resultados del ángulo de contacto del poliestireno reciclado, poliestireno comercial, los polímeros funcionalizados y sus híbridos correspondientes. Aquí podemos observar que en general el recubrimiento aumenta el ángulo de contacto permitiendo que el material tenga un comportamiento hidrofóbico con ángulos mayores a 400, como puede apreciarse en la fotografía 1, que muestra el comportamiento de las películas sobre el sustrato con el agua.

TABLA 1. Ángulo de contacto en sustrato de vidrio con recubrimiento.

\begin{tabular}{|c|c|c|}
\hline Recubrimiento & Pelicula original sin sustrato & Original con sustrato \\
\hline Sustrato & & 37.6262 \\
\hline PSR & 63.6669 & 67.5206 \\
\hline PSR-F & 76.6075 & 82.0304 \\
\hline HPSR & 74.7449 & 69.7435 \\
\hline HPSR-F & 20.5801 & 68.2995 \\
\hline PS & 82.1467 & 87.4552 \\
\hline PS-F & 53.2858 & 77.4712 \\
\hline HPS & 81.2538 & 81.5966 \\
\hline HPS-F & 58.5432 & 78.8049 \\
\hline
\end{tabular}

Fuente: Elaboración de los autores.

FOTOGRAFíA 1. Prueba de hidrofobicidad de las películas sobres sustrato de vidrio.

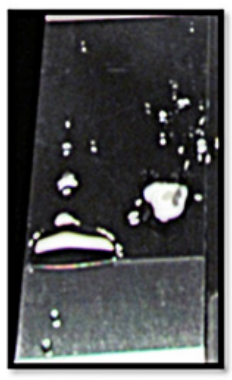

PS-F

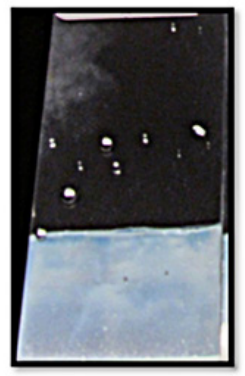

PSRF

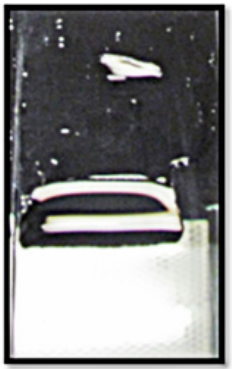

HPS

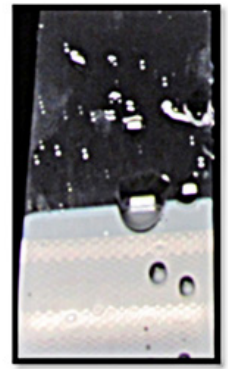

HPSRF

Fuente: Obtenidas por los autores. 


\section{Conclusión}

Se prepararon exitosamente materiales híbridos con poliestireno reciclado. Se introdujeron partículas de sílice a la matriz polimérica mediante el proceso sol-gel. La matriz polimérica fue modificada con ácido abiético, identificando mediante espectroscopía FT-IR el grupo caboxil de los polímeros funcionalizados (PSF y PRSF). El cual desaparece en los materiales híbridos (HPSF y HPSRF) lo cual sugiere una interacción del - $\mathrm{COOH}$ con los silanoles.

Por medio de SEM se observó que los híbridos de poliestireno (HPS y HPSR) muestran segregación de las partículas de sílice en la superficie de los materiales, en tanto los híbridos funcionalizados se observan más homogéneos, lo cual influye en la transparencia visual de los recubrimientos. Estos dos materiales híbridos también muestran un incremento en su Tg. La presencia de otros compuestos en el PSR, atribuidos a que el poliestireno es un material reciclado, esto no afecta el proceso de preparación ni las propiedades de hidrofobicidad y homogeneidad de los híbridos funcionalizados.

Los ángulos de contacto del poliestireno reciclado son muy similares al poliestireno comercial, obteniendo resultados satisfactorios de hidrofobicidad, por lo que estos recubrimientos pueden ser comercialmente interesantes ya que pueden aplicarse como recubrimientos autolimpiables, por ejemplo, en ventanas de edificios.

Los resultados de citotoxicidad muestran la viabilidad de usar las películas de poliestireno reciclado como recubrimientos sobre sustratos de vidrio, comparadas con sus análogas hechas con poliestireno comercial, lo cual abre una nueva alternativa para el reuso de este polímero basado en la metodología propuesta en este trabajo. Asimismo es factible que puedan ser fabricadas a nivel industrial.

\section{Referencias}

BaoLi, O. U. y L. (2007). DuXin, Preparation of polystyrene/silica nanocomposites by radical copolymerization of styrene with silica macromonomer. Sci. China Ser B-Chem., 50(3): 385-391.

Borsoia, C.; K. H. Berwigb, L. C. Scienzac, B. C. D. A. Zoppasd, R. N. Brandalisea, A. J. Zatteraa (2014). Behavior in simulated soil of recycled expanded polystyrene/waste cotton composites. Materials Research, 17(1): 275-283.

https://doi.org/10.1590/S1516-14392013005000167

Colthup, B., Daly H., Wiberley E. (1990). Introduction to infrared and Raman spectroscopy. 3rd. Academic Press, Sn. Diego Cal.

Gerasin, V. A.; E. M. Antipov, V. V. Karbushev, V. G. Kulichikhin, G. P. Karpacheva, R. V. Talroze, Y. V. Kudryavtsev (2013). New approaches to the development of hybrid nanocomposites: From structural materials to high-tech applications. Russian Chemical Reviews, 82(4): 303-332.

https://doi.org/10.1070/RC2013v082n04ABEH004322 
Hernández-Padrón, G.; F. Rojas, M. García-Garduño, M. A. Canseco y V. M. Castaño (2003). Development of hybrid materials consisting of $\mathrm{SiO}_{2}$ microparticles embedded in phenolic-formaldehydic resin polymer matrices. Mater. Sci. Eng. A, 355: 338-47. https://doi.org/10.1016/S0921-5093(03)00101-1

Hernández-Padrón, G.; M. García-Garduño y F. Rojas-González (2010). Hybrid materials based on functionalized epoxy resin netwoks. Pigment and Resin Technology, 39: 195-202. https://doi.org/10.1108/03699421011055491

Hernández-Padrón, Genoveva; Teresa Gómez Quintero y Alejandra Blanco Hernández (2014). Development antigfog nanohybrid consisting of polystyrene recycle matrix by sol-gel process. TechConnect World 2014, Nanotechnology Conferences and Expo-Nanotech 2014, Nanotech, 1, cap. 3: 218-219.

Hernández-Padrón, G.; Domingo Rangel-Miranda, Gerardo Cedillo y Alejandra Blanco-Hernández (2015). Incorporation of nanohybrid films of silica into recycled polystyrene matrix. Journal of Nanomaterials, vol. 2015.

https://doi.org/10.1155/2015/173949

Hossein, S. V.; A. Mohamadreza, N. Melsam, A. O. Iraj, S. Zahra, E. Mohamad (2011). Investigation of the fracture mechanism and mechanical properties of polystyrene/silica nancomposite in various silica contents. J. Mater. Sci., 46: 56285638. https://doi.org/10.1007/s10853-011-5513-9

Howarter, J. A.; J. P. Youngblood (2008). Self-cleaning and next generation anti-fog surfaces and coatings. Macromol. Rapid Commun., 29: 455-466.

https://doi.org/10.1002/marc.200700733

Hwang, D. R.; J. Hong, J. Lee y S. E. Shim (2008). In-situ synthesis of PS/(-)Silica composite particles in dispersion polymerization using an ( \pm ) amphoteric initiator. Macromolecular Research, 16(4): 329-336.

https://doi.org/10.1007/BF03218525

Ilgenenfritz, E. M. (1975). Plastics waste handling practices in soild waste management, Waste, Air and Soil Pollution, 4: 191-198.

Kotoky, T. y S. K. Dolui (2006). Synthesis of polystyrene/silica hybrid composites by the sol-gel method: Effect of introduction of a flexible component (butyl acrylate) into the silylated polystyrene backbone. Colloid Polym Sci., 284: 11631169. https://doi.org/10.1007/s00396-006-1498-3

Maharana, T.; Y. S. Negi y B. Mohanty (2014). Review article: Recicling of polystyrene. Polymer-Plastics Tecnology and Engineering, 46: 729-736. https://doi.org/10.1080/03602550701273963

Sánchez, C.; P. Belleville, M. Popall y L. Nicole (2011). Applications of advanced hybrid organic-inorganic nanomaterials: From laboratory to markets. Chemical Society Review, 40: 696-753. https://doi.org/10.1039/C0CS00136H

Stevenson, W.; J. R. White (2012). Photo-sensitivity of recycled photo-degraded polystyrene. J. Mater. Sci., 37: 1091-1100.

Zou, H.; W. Shishan y S. Jian (2008). Polymer/silica nanocomposites: Preparation, characterization, properties and applications. Chemical Reviews, 108: 38933957. https://doi.org/10.1021/cr068035q 
